\title{
Detection of the Characteristic Pion-decay Signature in Supernova Remnants
}

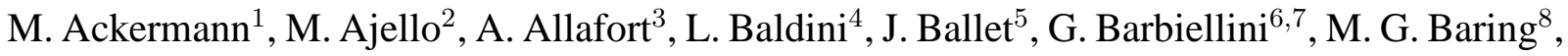 \\ D. Bastieri ${ }^{9,10}$, K. Bechtol ${ }^{3}$, R. Bellazzini ${ }^{11}$, R. D. Blandford ${ }^{3}$, E. D. Bloom ${ }^{3}$, E. Bonamente ${ }^{12,13}$, \\ A. W. Borgland ${ }^{3}$, E. Bottacini ${ }^{3}$, T. J. Brandt ${ }^{14}$, J. Bregeon ${ }^{11}$, M. Brigida ${ }^{15,16}$, P. Bruel $^{17}$, R. Buehler ${ }^{3}$, \\ G. Busetto ${ }^{9,10}$, S. Buson ${ }^{9,10}$, G. A. Caliandro ${ }^{18}$, R. A. Cameron ${ }^{3}$, P. A. Caraveo ${ }^{19}$, J. M. Casandjian ${ }^{5}$, \\ C. Cecchi ${ }^{12,13}$, Ö. Çelik ${ }^{14,20,21}$, E. Charles ${ }^{3}$, S. Chaty ${ }^{5}$, R.C.G. Chaves ${ }^{5}$, A. Chekhtman ${ }^{22}$, \\ C. C. Cheung ${ }^{23}$, J. Chiang ${ }^{3}$, G. Chiaro ${ }^{24}$, A. N. Cillis ${ }^{25,14}$, S. Ciprini ${ }^{26,13}$, R. Claus ${ }^{3}$, J. Cohen- \\ Tanugi $^{27}$, L. R. Cominsky ${ }^{28}$, J. Conrad ${ }^{29,30,31}$, S. Corbel ${ }^{5,32}$, S. Cutini ${ }^{33}$, F. D' Ammando ${ }^{12,34,35}$, \\ A. de Angelis ${ }^{36}$, F. de Palma ${ }^{15,16}$, C. D. Dermer ${ }^{37}$, E. do Couto e Silva ${ }^{3}$, P. S. Drell ${ }^{3}$, A. Drlica- \\ Wagner $^{3}$, L. Falletti ${ }^{27}$, C. Favuzzi ${ }^{15,16}$, E. C. Ferrara ${ }^{14}$, A. Franckowiak ${ }^{3}$, Y. Fukazawa ${ }^{38}$, S. Funk ${ }^{3 \star}$, \\ P. Fusco ${ }^{15,16}$, F. Gargano ${ }^{16}$, S. Germani ${ }^{12,13}$, N. Giglietto ${ }^{15,16}$, P. Giommi ${ }^{33}$, F. Giordano ${ }^{15,16}$, \\ M. Giroletti ${ }^{39}$, T. Glanzman ${ }^{3}$, G. Godfrey ${ }^{3}$, I. A. Grenier ${ }^{5}$, M.-H. Grondin ${ }^{40,41}$, J. E. Grove ${ }^{37}$, \\ S. Guiriec ${ }^{14}$, D. Hadasch ${ }^{18}$, Y. Hanabata ${ }^{38}$, A. K. Harding ${ }^{14}$, M. Hayashida $^{3,42}$, K. Hayashi ${ }^{38}$, \\ E. Hays ${ }^{14}$, J.W. Hewitt ${ }^{14}$, A. B. Hill ${ }^{3,43}$, R. E. Hughes ${ }^{44}$, M. S. Jackson ${ }^{45,30}$, T. Jogler ${ }^{3}$, G. Jóhannesson ${ }^{46}$, \\ A. S. Johnson ${ }^{3}$, T. Kamae ${ }^{3}$, J. Kataoka ${ }^{47}$, J. Katsuta ${ }^{3}$, J. Knödlseder ${ }^{48,49}$, M. Kuss ${ }^{11}$, J. Lande ${ }^{3}$, \\ S. Larsson ${ }^{29,30,50}$, L. Latronico ${ }^{51}$, M. Lemoine-Goumard ${ }^{52,53}$, F. Longo ${ }^{6,7}$, F. Loparco ${ }^{15,16}$, M. N. Lovellette ${ }^{37}$, \\ P. Lubrano ${ }^{12,13}$, G. M. Madejski ${ }^{3}$, F. Massaro ${ }^{3}$, M. Mayer ${ }^{1}$, M. N. Mazziotta ${ }^{16}$, J. E. McEnery ${ }^{14,54}$, \\ J. Mehault ${ }^{27}$, P. F. Michelson ${ }^{3}$, R. P. Mignani ${ }^{55}$, W. Mitthumsiri ${ }^{3}$, T. Mizuno ${ }^{56}$, A. A. Moiseev ${ }^{20,54}$, \\ M. E. Monzani ${ }^{3}$, A. Morselli ${ }^{57}$, I. V. Moskalenko ${ }^{3}$, S. Murgia ${ }^{3}$, T. Nakamori ${ }^{47}$, R. Nemmen ${ }^{14}$, \\ E. Nuss ${ }^{27}$, M. Ohno ${ }^{58}$, T. Ohsugi ${ }^{56}$, N. Omodei ${ }^{3}$, M. Orienti ${ }^{39}$, E. Orlando ${ }^{3}$, J. F. Ormes ${ }^{59}$, \\ D. Paneque ${ }^{60,3}$, J. S. Perkins ${ }^{14,21,20,61}$, M. Pesce-Rollins ${ }^{11}$, F. Piron ${ }^{27}$, G. Pivato ${ }^{10}$, S. Rainò ${ }^{15,16}$,

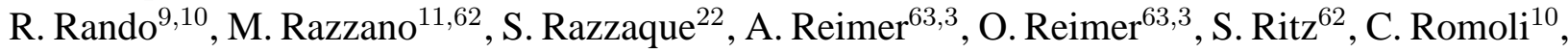 \\ M. Sánchez-Conde ${ }^{3}$, A. Schulz ${ }^{1}$, C. Sgrò ${ }^{11}$, P. E. Simeon ${ }^{3}$, E. J. Siskind ${ }^{64}$, D. A. Smith ${ }^{52}$, \\ G. Spandre ${ }^{11}$, P. Spinelli ${ }^{15,16}$, F. W. Stecker ${ }^{14,65}$, A. W. Strong ${ }^{66}$, D. J. Suson ${ }^{67}$, H. Tajima ${ }^{3,68}$, \\ H. Takahashi ${ }^{38}$, T. Takahashi ${ }^{58}$, T. Tanaka ${ }^{3,69 \dagger}$, J. G. Thayer ${ }^{3}$, J. B. Thayer ${ }^{3}$, D. J. Thompson ${ }^{14}$, \\ S. E. Thorsett ${ }^{70}$, L. Tibaldo ${ }^{9,10}$, O. Tibolla ${ }^{71}$, M. Tinivella ${ }^{11}$, E. Troja ${ }^{14,72}$, Y. Uchiyama ${ }^{3 \ddagger}$, \\ T. L. Usher ${ }^{3}$, J. Vandenbroucke ${ }^{3}$, V. Vasileiou ${ }^{27}$, G. Vianello ${ }^{3,73}$, V. Vitale ${ }^{57,74}$, A. P. Waite ${ }^{3}$,

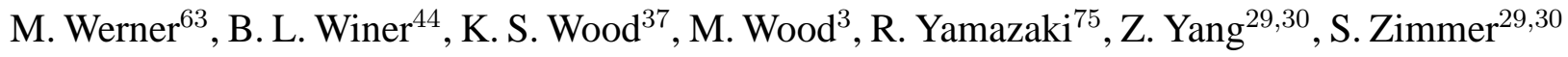

1. Deutsches Elektronen Synchrotron DESY, D-15738 Zeuthen, Germany 
2. Space Sciences Laboratory, 7 Gauss Way, University of California, Berkeley, CA 947207450, USA

3. W. W. Hansen Experimental Physics Laboratory, Kavli Institute for Particle Astrophysics and Cosmology, Department of Physics and SLAC National Accelerator Laboratory, Stanford University, Stanford, CA 94305, USA

4. Università di Pisa and Istituto Nazionale di Fisica Nucleare, Sezione di Pisa I-56127 Pisa, Italy

5. Laboratoire AIM, CEA-IRFU/CNRS/Université Paris Diderot, Service d'Astrophysique, CEA Saclay, 91191 Gif sur Yvette, France

6. Istituto Nazionale di Fisica Nucleare, Sezione di Trieste, I-34127 Trieste, Italy

7. Dipartimento di Fisica, Università di Trieste, I-34127 Trieste, Italy

8. Rice University, Department of Physics and Astronomy, MS-108, P. O. Box 1892, Houston, TX 77251, USA

9. Istituto Nazionale di Fisica Nucleare, Sezione di Padova, I-35131 Padova, Italy

10. Dipartimento di Fisica e Astronomia "G. Galilei", Università di Padova, I-35131 Padova, Italy

11. Istituto Nazionale di Fisica Nucleare, Sezione di Pisa, I-56127 Pisa, Italy

12. Istituto Nazionale di Fisica Nucleare, Sezione di Perugia, I-06123 Perugia, Italy

13. Dipartimento di Fisica, Università degli Studi di Perugia, I-06123 Perugia, Italy

14. NASA Goddard Space Flight Center, Greenbelt, MD 20771, USA

15. Dipartimento di Fisica "M. Merlin" dell'Università e del Politecnico di Bari, I-70126 Bari, Italy

16. Istituto Nazionale di Fisica Nucleare, Sezione di Bari, 70126 Bari, Italy

17. Laboratoire Leprince-Ringuet, École polytechnique, CNRS/IN2P3, 91128 Palaiseau, France

18. Institut de Ciències de l'Espai (IEEE-CSIC), Campus UAB, 08193 Barcelona, Spain

19. INAF-Istituto di Astrofisica Spaziale e Fisica Cosmica, I-20133 Milano, Italy

20. Center for Research and Exploration in Space Science and Technology (CRESST) and NASA Goddard Space Flight Center, Greenbelt, MD 20771, USA 
21. Department of Physics and Center for Space Sciences and Technology, University of Maryland Baltimore County, Baltimore, MD 21250, USA

22. Center for Earth Observing and Space Research, College of Science, George Mason University, Fairfax, VA 22030, resident at Naval Research Laboratory, Washington, DC 20375, USA

23. National Research Council Research Associate, National Academy of Sciences, Washington, DC 20001, resident at Naval Research Laboratory, Washington, DC 20375, USA

24. INFN and Dipartimento di Fisica e Astronomia "G. Galilei”, Università di Padova, I35131 Padova, Italy,

25. Instituto de Astronomía y Fisica del Espacio, Pabellón IAFE, Cdad. Universitaria, C1428ZAA, Buenos Aires, Argentina

26. ASI Science Data Center, I-00044 Frascati (Roma), Italy

27. Laboratoire Univers et Particules de Montpellier, Université Montpellier 2, CNRS/IN2P3, Montpellier, France

28. Department of Physics and Astronomy, Sonoma State University, Rohnert Park, CA 94928-3609, USA

29. Department of Physics, Stockholm University, AlbaNova, SE-106 91 Stockholm, Sweden

30. The Oskar Klein Centre for Cosmoparticle Physics, AlbaNova, SE-106 91 Stockholm, Sweden

31. Royal Swedish Academy of Sciences Research Fellow, funded by a grant from the K. A. Wallenberg Foundation

32. Institut universitaire de France, 75005 Paris, France

33. Agenzia Spaziale Italiana (ASI) Science Data Center, I-00044 Frascati (Roma), Italy

34. IASF Palermo, 90146 Palermo, Italy

35. INAF-Istituto di Astrofisica Spaziale e Fisica Cosmica, I-00133 Roma, Italy

36. Dipartimento di Fisica, Università di Udine and Istituto Nazionale di Fisica Nucleare, Sezione di Trieste, Gruppo Collegato di Udine, I-33100 Udine, Italy

37. Space Science Division, Naval Research Laboratory, Washington, DC 20375-5352, USA

38. Department of Physical Sciences, Hiroshima University, Higashi-Hiroshima, Hiroshima 739-8526, Japan 
39. INAF Istituto di Radioastronomia, 40129 Bologna, Italy

40. Max-Planck-Institut für Kernphysik, D-69029 Heidelberg, Germany

41. Landessternwarte, Universität Heidelberg, Königstuhl, D 69117 Heidelberg, Germany

42. Department of Astronomy, Graduate School of Science, Kyoto University, Sakyo-ku, Kyoto 606-8502, Japan

43. School of Physics and Astronomy, University of Southampton, Highfield, Southampton, SO17 1BJ, UK

44. Department of Physics, Center for Cosmology and Astro-Particle Physics, The Ohio State University, Columbus, OH 43210, USA

45. Department of Physics, Royal Institute of Technology (KTH), AlbaNova, SE-106 91 Stockholm, Sweden

46. Science Institute, University of Iceland, IS-107 Reykjavik, Iceland

47. Research Institute for Science and Engineering, Waseda University, 3-4-1, Okubo, Shinjuku, Tokyo 169-8555, Japan

48. CNRS, IRAP, F-31028 Toulouse cedex 4, France

49. GAHEC, Université de Toulouse, UPS-OMP, IRAP, 31028 Toulouse, France

50. Department of Astronomy, Stockholm University, SE-106 91 Stockholm, Sweden

51. Istituto Nazionale di Fisica Nucleare, Sezione di Torino, I-10125 Torino, Italy

52. Université Bordeaux 1, CNRS/IN2p3, Centre d'Études Nucléaires de Bordeaux Gradignan, 33175 Gradignan, France

53. Funded by contract ERC-StG-259391 from the European Community

54. Department of Physics and Department of Astronomy, University of Maryland, College Park, MD 20742, USA

55. Mullard Space Science Laboratory, University College London, Holmbury St. Mary, Dorking, Surrey, RH5 6NT, UK

56. Hiroshima Astrophysical Science Center, Hiroshima University, Higashi-Hiroshima, Hiroshima 739-8526, Japan

57. Istituto Nazionale di Fisica Nucleare, Sezione di Roma "Tor Vergata", I-00133 Roma, Italy 
58. Institute of Space and Astronautical Science, JAXA, 3-1-1 Yoshinodai, Chuo-ku, Sagamihara, Kanagawa 252-5210, Japan

59. Department of Physics and Astronomy, University of Denver, Denver, CO 80208, USA

60. Max-Planck-Institut für Physik, D-80805 München, Germany

61. Harvard-Smithsonian Center for Astrophysics, Cambridge, MA 02138, USA

62. Santa Cruz Institute for Particle Physics, Department of Physics and Department of Astronomy and Astrophysics, University of California at Santa Cruz, Santa Cruz, CA 95064, USA

63. Institut für Astro- und Teilchenphysik and Institut für Theoretische Physik, LeopoldFranzens-Universität Innsbruck, A-6020 Innsbruck, Austria

64. NYCB Real-Time Computing Inc., Lattingtown, NY 11560-1025, USA

65. Department of Physics and Astronomy, University of California, Los Angeles, CA 900951547, USA

66. Max-Planck Institut für extraterrestrische Physik, 85748 Garching, Germany

67. Department of Chemistry and Physics, Purdue University Calumet, Hammond, IN 463232094, USA

68. Solar-Terrestrial Environment Laboratory, Nagoya University, Nagoya 464-8601, Japan

69. Department of Physics, Graduate School of Science, Kyoto University, Sakyo-ku, Kyoto 606-8502, Japan

70. Department of Physics, Willamette University, Salem, OR 97031, USA

71. Institut für Theoretische Physik and Astrophysik, Universität Würzburg, D-97074 Würzburg, Germany

72. NASA Postdoctoral Program Fellow

73. Consorzio Interuniversitario per la Fisica Spaziale, I-10133 Torino, Italy

74. Dipartimento di Fisica, Università di Roma “Tor Vergata”, I-00133 Roma, Italy

75. Department of Physics and Mathematics, Aoyama Gakuin University, Sagamihara, Kanagawa, 252-5258, Japan

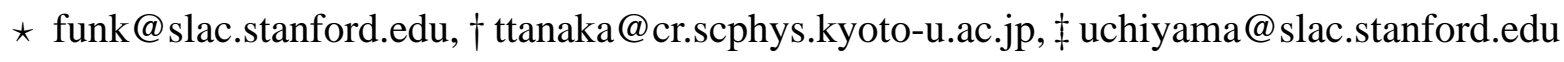


Cosmic rays are particles (mostly protons) accelerated to relativistic speeds. Despite wide agreement that supernova remnants (SNRs) are the sources of galactic cosmic rays, unequivocal evidence for the acceleration of protons in these objects is still lacking. When accelerated protons encounter interstellar material they produce neutral pions, which in turn decay into gamma rays. This offers a compelling way to detect the acceleration sites of protons. The identification of pion-decay gamma rays has been difficult because high-energy electrons also produce gamma rays via bremsstrahlung and inverse Compton scattering. We detected the characteristic pion-decay feature in the gammaray spectra of two SNRs, IC 443 and W44, with the Fermi Large Area Telescope. This detection provides direct evidence that cosmic-ray protons are accelerated in SNRs.

A supernova explosion drives its progenitor material supersonically into interstellar space, forming a collisionless shock wave ahead of the stellar ejecta. The huge amount of kinetic energy released by a supernova, typically $10^{51} \mathrm{ergs}$, is initially carried by the expanding ejecta and is then transferred to kinetic and thermal energies of shocked interstellar gas and relativistic particles. The shocked gas and relativistic particles produce the thermal and nonthermal emissions of a supernova remnant (SNR). The mechanism of diffusive shock acceleration (DSA) can explain the production of relativistic particles in SNRs (1). DSA generally predicts that a substantial fraction of the shock energy is transferred to relativistic protons. Indeed, if SNRs are the main sites of acceleration of the galactic cosmic rays, then 3 to $30 \%$ of the supernova kinetic energy must end up transferred to relativistic protons. However, the presence of relativistic protons in SNRs has been mostly inferred from indirect arguments (2-5).

A direct signature of high energy protons is provided by gamma rays generated in the decay of neutral pions $\left(\pi^{0}\right)$; proton-proton (more generally nuclear-nuclear) collisions create $\pi^{0}$ mesons which usually quickly decay into two gamma rays (6-8) (schematically written as $p+p \rightarrow \pi^{0}+$ other products, followed by $\pi^{0} \rightarrow 2 \gamma$ ), each having an energy of $m_{\pi^{0}} c^{2} / 2=67.5$ $\mathrm{MeV}$ in the rest frame of the neutral pion (where $m_{\pi^{0}}$ is the rest mass of the neutral pion and $c$ is the speed of light). The gamma-ray number spectrum, $F(\varepsilon)$, is thus symmetric about $67.5 \mathrm{MeV}$ in a $\log$-log representation (9). The $\pi^{0}$-decay spectrum in the usual $\varepsilon^{2} F(\varepsilon)$ representation rises steeply below $\sim 200 \mathrm{MeV}$ and approximately traces the energy distribution of parent protons at energies greater than a few $\mathrm{GeV}$. This characteristic spectral feature (often referred to as the "pion-decay bump") uniquely identifies $\pi^{0}$-decay gamma rays and thereby high-energy protons, allowing a measurement of the source spectrum of cosmic rays.

Massive stars are short-lived and end their lives with core-collapse supernova explosions. These explosions typically occur in the vicinity of molecular clouds with which they interact. When cosmic-ray protons accelerated by SNRs penetrate into high density clouds, $\pi^{0}$-decay gamma-ray emission is expected to be enhanced because of more frequent $p p$ interactions relative to the interstellar medium (10). Indeed, SNRs interacting with molecular clouds are the most luminous SNRs in gamma rays $(11,12)$. The best examples of SNR-cloud interactions in 
our galaxy are the SNRs IC 443 and W44 (13), which are the two highest-significance SNRs in the second Fermi Large Area Telescope (LAT) catalog (2FGL) (14) and are thus particularly suited for a dedicated study of the details of their gamma-ray spectra. The age of each remnant is estimated to be $\sim 10,000$ years. IC 443 and W44 are located at distances of $1.5 \mathrm{kpc}$ and 2.9 kpc, respectively.

We report here on 4 years of observations with the Fermi LAT (4 August 2008 to 16 July 2012) of IC 443 and W44, focusing on the sub-GeV part of the gamma-ray spectrum - a crucial spectral window for distinguishing $\pi^{0}$-decay gamma rays from electron bremsstrahlung or inverse Compton scattering produced by relativistic electrons. Previous analyses of IC 443 and W44 used only 1 year of Fermi LAT data (15-17) and were limited to the energy band above $200 \mathrm{MeV}$, mainly because of the small and rapidly changing LAT effective area at low energies. A recent update to the event classification and background rejection (so-called Pass 7) provides an increase in LAT effective area at $100 \mathrm{MeV}$ by a factor of $\sim 5$ (18), enabling the study of bright, steady sources in the galactic plane below $200 \mathrm{MeV}$ with the Fermi-LAT. Note that the gamma-ray spectral energy distribution of W44 measured recently by the AGILE satellite falls steeply below $1 \mathrm{GeV}$, which the authors interpreted as a clear indication for the $\pi^{0}$-decay origin of the gamma-ray emission (19). Also, a recent analysis of W44 at high energies (above $2 \mathrm{GeV}$ ) has been reported (20), revealing large-scale gamma-ray emission attributable to high-energy protons that have escaped from W44. Here we present analyses of the gamma-ray emission from the compact regions delineated by the radio continuum emission of IC 443 and W44.

The analysis was performed using the Fermi LAT Science Tools (21). We used a maximum likelihood technique to determine the significance of a source over the background and to fit spectral parameters $(22,23)$. For both SNRs, additional sources seen as excesses in the background-subtracted map have been added to the background model (24) and are shown as diamonds in Fig. 1 - one in the case of IC 443, three in the case of W44. The inclusion of these sources had no influence on the fitted spectrum of the SNRs. Three close-by sources (2FGL J1852.8+0156c, 2FGL J1857.2+0055c, and 2FGL J1858.5+0129c) have been identified with escaping cosmic rays from W44 (20). These 2FGL sources have been removed from the background model (see below) in order to measure the full cosmic-ray content of W44.

Figure 2 shows the spectral energy distribution obtained for IC 443 and W44 through maximum likelihood estimation. To derive the flux points we performed a maximum likelihood fitting in 24 independent logarithmically spaced energy bands from $60 \mathrm{MeV}$ to $100 \mathrm{GeV}$. The normalization of the fluxes of IC 443 and W44 and those of neighboring sources and of the galactic diffuse model, was left free in the fit for each bin. In both sources, the spectra below $\sim 200 \mathrm{MeV}$ are steeply rising, clearly exhibiting a break at $\sim 200 \mathrm{MeV}$. To quantify the significances of the spectral breaks, we fit the fluxes of IC 443 and W44 between $60 \mathrm{MeV}$ and 2 $\mathrm{GeV}$ - below the high-energy breaks previously found in the 1-year spectra $(15,16)$ with both a single power law of the form $F(\varepsilon)=K\left(\varepsilon / \varepsilon_{0}\right)^{-\Gamma_{1}}$ and a smoothly broken power law of the form $F(\varepsilon)=K\left(\varepsilon / \varepsilon_{0}\right)^{-\Gamma_{1}}\left(1+\left(\varepsilon / \varepsilon_{\mathrm{br}}\right)^{\left(\Gamma_{2}-\Gamma_{1}\right) / \alpha}\right)^{-\alpha}$ with $\varepsilon_{0}=200 \mathrm{MeV}$. The spectral index changes from $\Gamma_{1}$ to $\Gamma_{2}\left(>\Gamma_{1}\right)$ at the break energy $\varepsilon_{\mathrm{br}}$. The smoothness of the break is determined by the parameter $\alpha$, which was fixed at 0.1 (Table 1). We define the test-statistic value 
(TS) as $2 \ln \left(\mathcal{L}_{1} / \mathcal{L}_{0}\right)$ where $\mathcal{L}_{1 / 0}$ corresponds to the likelihood value for the source/no-source hypothesis (23). The detection significance is given by $\sim \sqrt{T S}$. The smoothly broken power law model yields a significantly larger $T S$ than a single power law, establishing the existence of a low-energy break. The improvement in log likelihood when comparing the broken power law to a single power law corresponds to a formal statistical significance of $19 \sigma$ for the low-energy break in IC 443 and $21 \sigma$ for that in W44, when assuming a nested model with two additional degrees of freedom.

Table 1: Spectral parameters in the energy range of $60 \mathrm{MeV}$ to $2 \mathrm{GeV}$ for power-law (PL) and broken power-law (BPL) models. $T S=2 \ln \left(\mathcal{L}_{1} / \mathcal{L}_{2}\right)$ is the test-statistic value.

\begin{tabular}{cccccc}
\hline Model & $K\left(\mathrm{~cm}^{2} \mathrm{~s}^{-1} \mathrm{MeV}^{-1}\right)$ & $\Gamma_{1}$ & $\Gamma_{2}$ & $\varepsilon_{\mathrm{br}}(\mathrm{MeV})$ & $T S$ \\
\hline IC 443 & & & & & \\
PL & $11.7 \pm 0.2) \times 10^{-10}$ & $1.76 \pm 0.02$ & $\ldots$ & $\ldots$ & 21651 \\
BPL & $(11.9 \pm 0.6) \times 10^{-10}$ & $0.57 \pm 0.25$ & $1.95_{-0.02}^{+0.02}$ & $245_{-15}^{+16}$ & 22010 \\
W44 & & & & & \\
PL & $(13.0 \pm 0.4) \times 10^{-10}$ & $1.71 \pm 0.03$ & $\ldots$ & $\ldots$ & 6920 \\
BPL & $(15.8 \pm 1.0) \times 10^{-10}$ & $0.07 \pm 0.4$ & $2.08_{-0.03}^{+0.03}$ & $253_{-11}^{+11}$ & 7351 \\
\hline
\end{tabular}

To determine whether the spectral shape could indeed be modeled with accelerated protons, we fit the LAT spectral points with a $\pi^{0}$-decay spectral model, which was numerically calculated from a parameterized energy distribution of relativistic protons. Following previous studies $(15,16)$, the parent proton spectrum as a function of momentum $p$ was parameterized by a smoothly broken power law in the form of

$$
\frac{d N_{p}}{d p} \propto p^{-s_{1}}\left[1+\left(\frac{p}{p_{\mathrm{br}}}\right)^{\frac{s_{2}-s_{1}}{\beta}}\right]^{-\beta} .
$$

Best-fit parameters were searched using $\chi^{2}$-fitting to the flux points. The measured gamma-ray spectra, in particular the low-energy parts, matched the $\pi^{0}$-decay model (Figure 2). Parameters for the underlying proton spectrum are $s_{1}=2.36 \pm 0.02, s_{2}=3.1 \pm 0.1$, and $p_{\mathrm{br}}=239 \pm$ $74 \mathrm{GeV} c^{-1}$ for IC 443 and $s_{1}=2.36 \pm 0.05, s_{2}=3.5 \pm 0.3$, and $p_{\mathrm{br}}=22 \pm 8 \mathrm{GeV} c^{-1}$ for W44 (statistical errors only). In Figure 3 we show the energy distributions of the high-energy protons derived from the gamma-ray fits. The break $p_{\text {br }}$ is at higher energies and is unrelated to the low-energy pion-decay bump seen in the gamma-ray spectrum. If the interaction between a cosmic-ray precursor (i.e., cosmic rays distributed in the shock upstream on scales smaller than $\sim 0.1 R$, where $R$ is the SNR radius) and adjacent molecular clouds were responsible for the bulk of the observed $\mathrm{GeV}$ gamma rays, one would expect a much harder energy spectrum at low energies (i.e. a smaller value for the index $s_{1}$ ), contrary to the Fermi observations. Presumably, cosmic raysin the shock downstream produce the observed gamma rays; the first 
index $s_{1}$ represents the shock-acceleration index with possible effects due to energy-dependent propagation, and $p_{\text {br }}$ may indicate the momentum above which protons cannot be effectively confined within the SNR shell. Note that $p_{\text {br }}$ results in the high-energy break in the gamma-ray spectra at $\sim 20 \mathrm{GeV}$ and $\sim 2 \mathrm{GeV}$ for IC 443 and W44, respectively.

The $\pi^{0}$-decay gamma rays are likely emitted through interactions between "crushed cloud" gas and relativistic protons, both of which are highly compressed by radiative shocks driven into molecular clouds that are overtaken by the blast wave of the SNR (25). Filamentary structures of synchrotron radiation seen in a high-resolution radio continuum map of W44 (26) support this picture. High-energy particles in the "crushed cloud" can be explained by re-acceleration of the pre-existing galactic cosmic rays (25) and/or freshly accelerated particles that have entered the dense region (20). The mass of the shocked gas $\left(\sim 1 \times 10^{3} M_{\odot}\right.$ and $\sim 5 \times 10^{3} M_{\odot}$ for IC 443 and W44 respectively, where $M_{\odot}$ is the mass of the Sun) is large enough to explain the observed gamma-ray luminosity. Because the "crushed cloud" is geometrically thin, multi-GeV particles are prone to escape from the dense gas, which may explain the break $p_{\mathrm{br}}$.

Escaped CRs reaching the unshocked molecular clouds ahead of the SNR shock can also produce $\pi^{0}$-decay gamma rays $(27,28)$. Indeed, the gamma rays emitted by the escaped CRs in the large molecular complex that surrounds W44 (total extent of $100 \mathrm{pc}$ ) have been identified with three close-by sources (20), which led us to remove them from the model in the maximum likelihood analysis, as mentioned above. With this treatment, the measured fluxes below $1 \mathrm{GeV}$ contain small contributions from the escaped CRs, but this does not affect our conclusions. The escaped CRs may significantly contribute to the measured TeV fluxes from IC $443(29,30)$. Emission models could be more complicated. For example, the CR precursor with a scale of $\sim 0.1 R$ at the highest energy could interact with the adjacent unshocked molecular gas, producing hard gamma-ray emission. This effect is expected to become important above the LAT energy range.

We should emphasize that radiation by relativistic electrons can not as naturally account for the gamma-ray spectra of the SNRs (24). An inverse-Compton origin of the emission was not plausible on energetic grounds (11). The most important seed photon population for scattering is the infrared radiation produced locally by the SNR itself with an energy density of $\sim 1 \mathrm{eV} \mathrm{cm}{ }^{-3}$, but this is not large enough to explain the observed gamma-ray emission. Unless we introduce in an ad hoc way an additional abrupt break in the electron spectrum at $300 \mathrm{MeV} c^{-1}$ (Fig. 2 dash-dotted lines), the bremsstrahlung models do not fit the observed gamma-ray spectra. If we assume that the same electrons are responsible for the observed synchrotron radiation in the radio band, a low-energy break is not expected to be very strong in the radio spectrum and thus the existing data do not rule out this scenario. The introduction of the low-energy break introduces additional complexity and therefore a bremsstrahlung origin is not preferred. Although most of the gamma-ray emission from these SNRs is due to $\pi^{0}$-decay, electron bremsstrahlung may still contribute at a lower level. The Fermi LAT data allow an electron-to-proton ratio $K_{e p}$ of $\sim 0.01$ or less, where $K_{e p}$ is defined as the ratio of $d N_{e} / d p$ and $d N_{p} / d p$ at $p=1 \mathrm{GeV} c^{-1}$ (figs. S2 and $[33$.

Finding evidence for the acceleration of protons has long been a key issue in attempts to elu- 
cidate the origin of cosmic rays. Our spectral measurements down to $60 \mathrm{MeV}$ enable the identification of the $\pi^{0}$-decay feature, thus providing direct evidence for the acceleration of protons in SNRs. The proton momentum distributions, well-constrained by the observed gamma-ray spectra, are yet to be understood in terms of acceleration and escape processes of high-energy particles.

\section{References and Notes}

1. M. A. Malkov, \& L. O’C. Drury, Reports on Progress in Physics 64, 429 (2001).

2. J. S. Warren, et al., Astrophys. J. 634, 276 (2005).

3. Y. Uchiyama, F. A. Aharonian, T. Tanaka, T. Takahashi, Y. Maeda, Nature 449, 576 (2007).

4. E. A. Helder, et al., Science 325, 719 (2009).

5. G. Morlino, D. Caprioli, Astron. Astrophys. 538, A81 (2012).

6. C. D. Dermer, Astron. Astrophys. 157, 223 (1986).

7. L. O’C. Drury, F. A. Aharonian, H. J. Völk, Astron. Astrophys. 287, 959 (1994).

8. T. Naito, F. Takahara, J. Phys. G 20, 477 (1994).

9. F. W. Stecker, Cosmic gamma rays, NASA special publication 249 (1971).

10. F. A. Aharonian, L. O’C Drury, H. J. Völk, Astron. Astrophys. 285, 645 (1994).

11. A. A. Abdo, et al., Astrophys. J. 706, L1 (2009).

12. D. J. Thompson, L. Baldini, Y. Uchiyama, Astropart. Phys. in press, arXiv:1201.0988.

13. M. Seta, et al., Astrophys. J. 505, 286 (1998).

14. P. L. Nolan, et al., Astrophys. J. Suppl. 199, 31 (2012).

15. A. A. Abdo, et al., Science 327, 1103 (2010).

16. A. A. Abdo, et al., Astrophys. J. 712, 459 (2010).

17. J. Lande, et al., Astrophys. J. 756, 5 (2012).

18. M. Ackermann et al., Astrophys. J. Suppl 203, 4 (2012)

19. A. Giuliani, et al., Astrophys. J. 742, L30 (2011).

20. Y. Uchiyama, et al., Astrophys. J. 749, L35 (2012).

21. http://fermi.gsfc.nasa.gov/ssc/

22. The region model fitted to the data includes the SNR of interest (IC 443 or W44), background point sources from the 2FGL catalog (14), the Galactic diffuse emission (ring_2year_P76_v0.fits) and a corresponding isotropic component (isotrop_2year_P76_source v0.txt).

23. J. R. Mattox, et al., Astrophys. J. 461, 396 (1996).

24. see Supplementary Online Material (SOM) for details

25. Y. Uchiyama, R. D. Blandford, S. Funk, H. Tajima, T. Tanaka, Astrophys. J. 723, L122 (2010).

26. G. Castelletti, G. Dubner, C. Brogan, C., N. E. Kassim, Astron. Astrophys. 471, 537 (2007).

27. S. Gabici, F. A. Aharonian, S. Casanova, Mon. Not. R. Astron. Soc. 396, 1629 (2009). 
28. Y. Ohira, K. Murase, R. Yamazaki, Mon. Not. R. Astron. Soc. 410, 1577 (2011).

29. J. Albert, et al., Astrophys. J. 664, L87 (2007).

30. V. A. Acciari, et al., Astrophys. J. 698, L133 (2009).

31. M. Tavani, et al., Astrophys. J. 710, L151 (2010).

32. T. Kamae, N. Karlsson, T. Mizuno, T. Abe, T. Koi, Astrophys. J. 647, 692 (2006).

33. M. Mori, Astropart. Phys., 31, 341 (2009)

34. R. Buehler, et al., Astrophys. J.749, 26 (2012).

35. A. W. Strong, I. V. Moskalenko, Astrophys. J. 509, 212 (1998).

36. M. Ackermann, et al., Astrophys. J. 750, 3 (2012).

37. E. Troja, F. Bocchino, M. Miceli, F. Reale,Astron. Astrophys. 485, 777 (2008).

38. W. T. Reach, J. Rho, T. H. Jarrett, Astrophys. J. 618, 297 (2005).

39. G. Castelletti, G. Dubner, T. Clarke, C., N. E. Kassim, Astron. Astrophys. 534, A21 (2011)

The Fermi LAT Collaboration acknowledges support from a number of agencies and institutes for both development and the operation of the LAT as well as scientific data analysis. These include NASA and the U.S. Department of Energy (United States); CEA/Irfu and IN2P3/CNRS (France); ASI and INFN (Italy); MEXT, KEK, and JAXA (Japan); and the K. A. Wallenberg Foundation, the Swedish Research Council and the National Space Board (Sweden). Additional support from INAF in Italy and CNES in France for science analysis during the operations phase is also gratefully acknowledged. Fermi LAT data are available from the Fermi Science Support Center (http://fermi.gsfc.nasa.gov/ssc). 


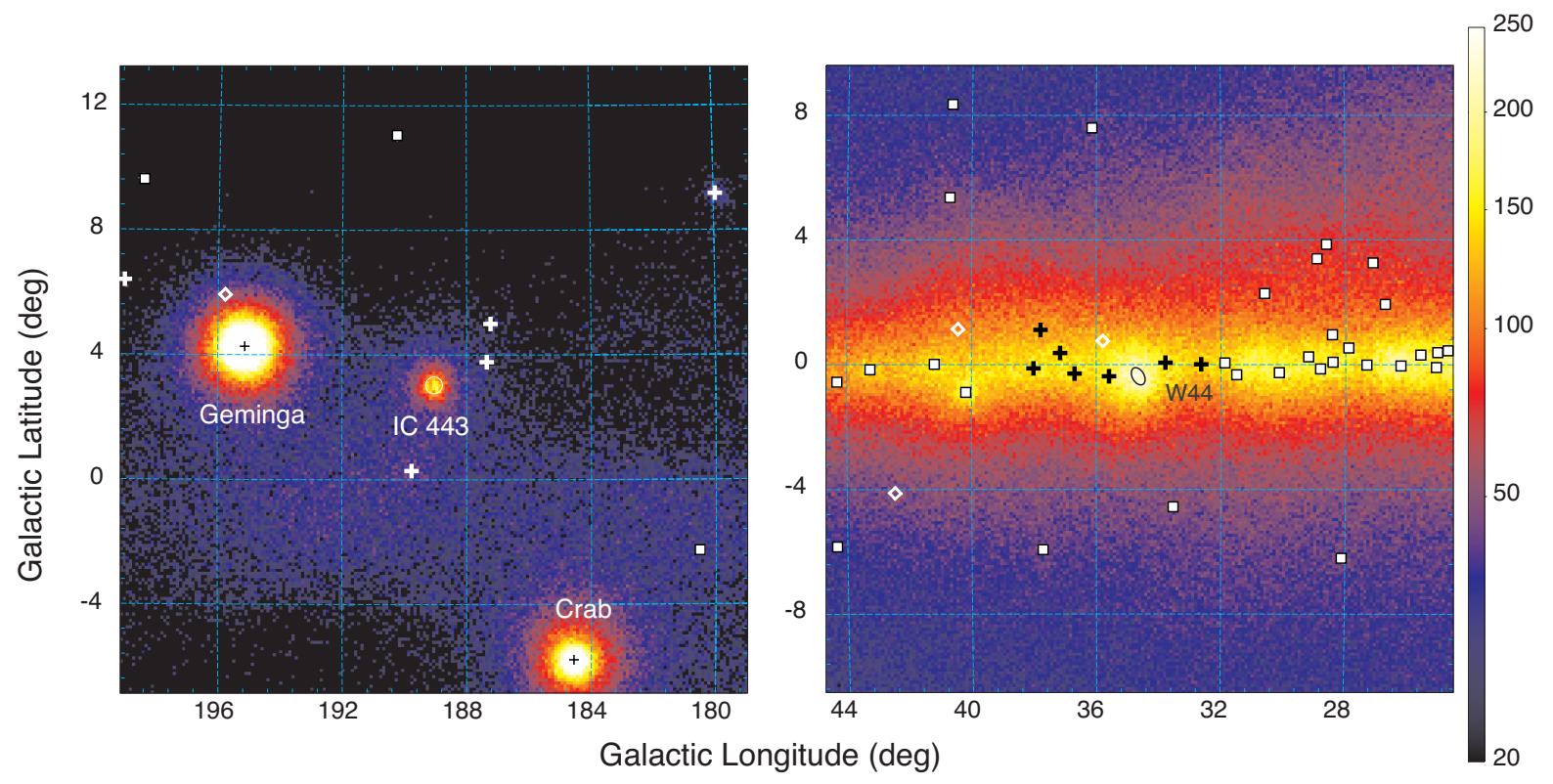

Figure 1: Gamma-ray count maps of the $20^{\circ} \times 20^{\circ}$ fields around IC 443 (left panel) and W44 (right panel) in the energy range $60 \mathrm{MeV}$ to $2 \mathrm{GeV}$. Nearby gamma-ray sources are marked as crosses and squares. Diamonds denote previously undetected sources. For sources indicated by crosses and diamonds, the fluxes were left as free parameters in the analysis. Events were spatially binned in regions of side length $0.1^{\circ}$, the units of the color bar is the square root of count density, and the colors have been clipped at 20 counts per pixel to make the galactic diffuse emission less prominent. Given the spectra of the sources and the effective area of the LAT instrument, the bulk of the photons seen in this plot have energies between 300 and 500 $\mathrm{MeV}$. IC 443 is located in the galactic anti-center region, where the background gamma-ray emission produced by the pool of galactic cosmic rays interacting with interstellar gas is rather weak relative to the region around W44. The two dominant sources in the IC 443 field are the Geminga pulsar (2FGL J0633.9+1746) and the Crab (2FGL J0534.5+2201). For the W44 count map, W44 is the dominant source, sub-dominant, however, to the galactic diffuse emission. 

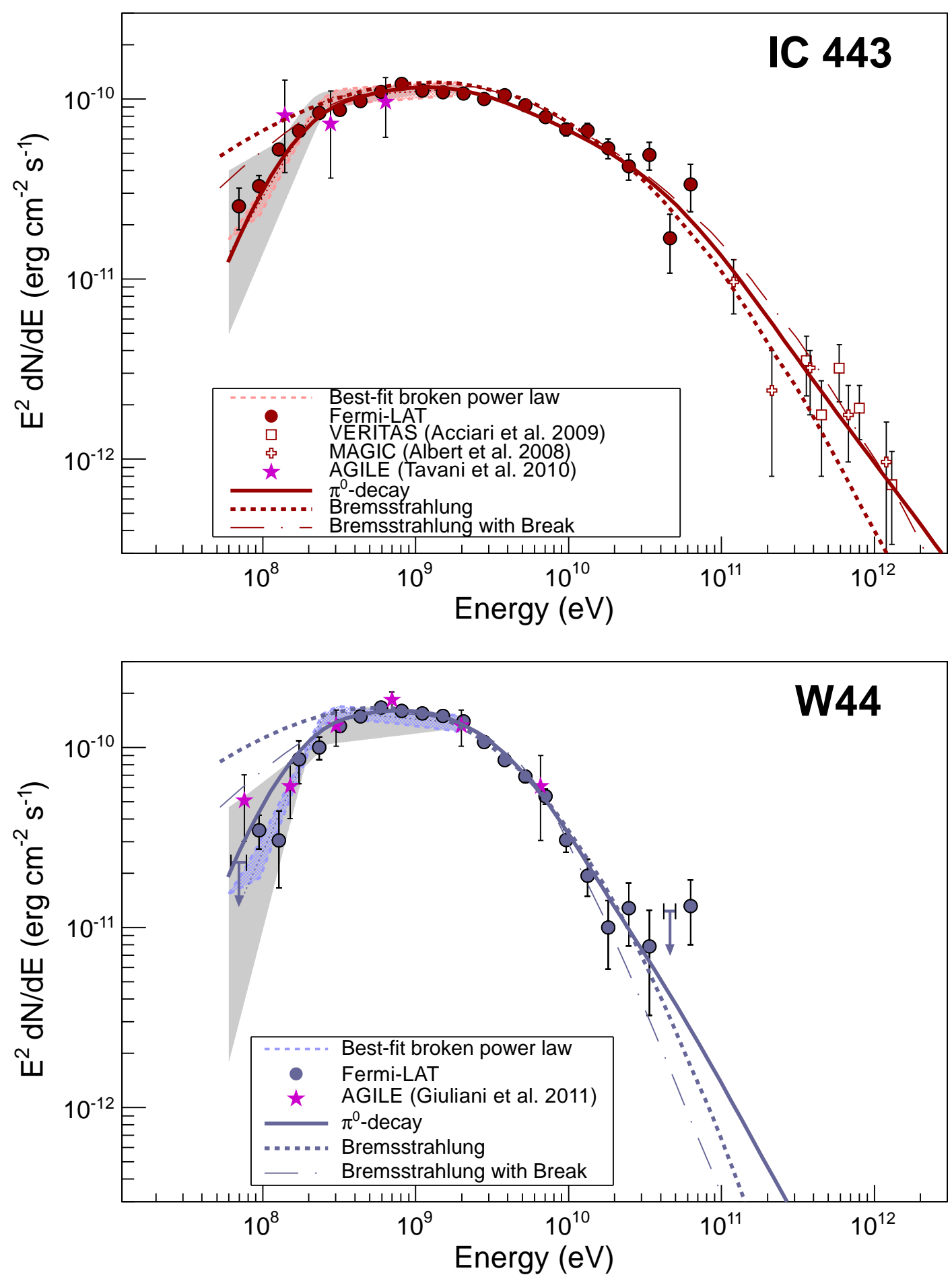

Figure 2: (A and B) Gamma-ray spectra of IC 443 (A) and W44 (B) as measured with the Fermi-LAT. Color-shaded areas bound by dashed lines denote the best-fit broadband smooth broken power law (60 MeV to $2 \mathrm{GeV}$ ), gray-shaded bands show systematic errors below 2 $\mathrm{GeV}$ due mainly to imperfect modeling of the galactic diffuse emission. At the high-energy end, TeV spectral data points for IC 443 from MAGIC (29) and VERITAS (30) are shown. Solid lines denote the best-fit pion-decay gamma-ray spectra, dashed lines denote the best-fit bremsstrahlung spectra, and dash-dotted lines denote the best-fit bremsstrahlung spectra when including an ad hoc low-energy break at $300 \mathrm{MeV} \mathrm{c} c^{-1}$ in the electron spectrum. These fits were done to the Fermi LAT data alone (not taking the TeV data points into account). Magenta stars denote measurements from the AGILE satellite for these two SNRs, taken from (31) and (19), respectively. 


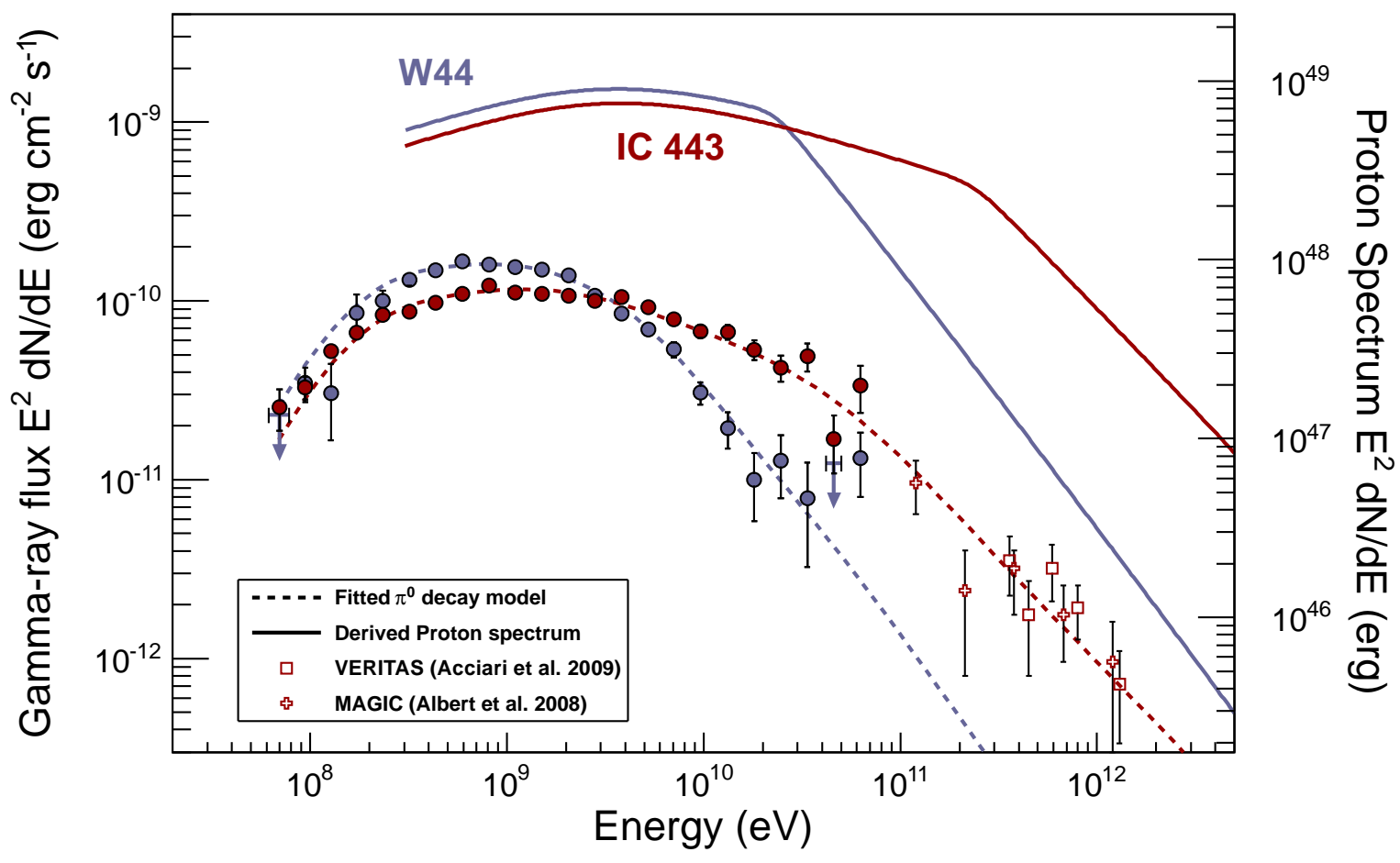

Figure 3: Proton and gamma-ray spectra determined for IC 443 and W44. Also shown are the broadband spectral flux points derived in this study, along with $\mathrm{TeV}$ spectral data points for IC 443 from MAGIC (29) and VERITAS (30). The curvature evident in the proton distribution at $\sim 2 \mathrm{GeV}$ is a consequence of the display in energy space (rather than momentum space). Gamma-ray spectra from the protons were computed using the energy-dependent cross section parameterized by (32). We took into account accelerated nuclei (heavier than protons) as well as nuclei in the target gas by applying an enhancement factor of 1.85 (33). Note that models of the gamma-ray production via $p p$ interactions have some uncertainty. Relative to the model adopted here, an alternative model of (6) predicts $\sim 30 \%$ less photon flux near $70 \mathrm{MeV}$; the two models agree with each other to better than $15 \%$ above $200 \mathrm{MeV}$. The proton spectra assume average gas densities of $n=20 \mathrm{~cm}^{-3}$ (IC 443) and $n=100 \mathrm{~cm}^{-3}$ (W44) and distances of 1.5 $\mathrm{kpc}$ (IC 443) and $2.9 \mathrm{kpc}$ (W44). 


\section{Supplementary Material}

\section{Analysis of the LAT data}

In its normal mode, the LAT scans the whole sky every 3 hours (two orbits). In this analysis, data taken in the sky-survey mode starting from the beginning of scientific operation on 2008 August 4 to 2012 July 16 were used. These data were analyzed using the LAT Science Tools package (v9r29), which is available from the Fermi Science Support Center. The analysis used the P7v6 version of the instrument response functions which take into account accidental coincidence effects in the detectors. Only events passing the "Source" class cuts are used in the analysis, and events coming from zenith angles $>100^{\circ}$ were discarded to reduce the contribution from the emission in the Earth's upper atmosphere. To further reduce these Earth-emitted gamma rays, intervals when the Earth was in the field of view were excluded, specifically when the rocking angle of the LAT was greater than $52^{\circ}$ or when parts of the region-of-interest (ROI) were observed at zenith angles $>100^{\circ}$. All gamma rays with energies $>60 \mathrm{MeV}$ within a $20^{\circ} \times 20^{\circ}$ region around the nominal positions of the sources were used.

During the broad-band fit of the source of interest, all 2FGL sources within the field of view were part of the likelihood model with the spectral models as adopted in the 2FGL catalog (14). Because the 2FGL catalog corresponds to 2 years of data, while the dataset used in the study comprises 4 years, we searched for additional (faint) sources within the ROI by constructing maps of residual significance after subtracting all known sources in the field. For the W44 region three additional sources were identified at positions $\left(\alpha_{\mathrm{J} 2000}, \delta_{\mathrm{J} 2000}\right)=\left(283.92^{\circ}, 2.79^{\circ}\right)$ with a TS value above $60 \mathrm{MeV}$ of $137,\left(285.25^{\circ}, 7.11^{\circ}\right)$ with a TS value of 98 , and $\left(290.90^{\circ}, 6.62^{\circ}\right)$ with a TS value of 52 , where $\alpha_{\mathrm{J} 2000}$ and $\delta_{\mathrm{J} 2000}$ are right ascension and declination for the epoch 2000 . In the IC 443 ROI one additional source was identified at $\left(\alpha_{\mathrm{J} 2000}, \delta_{\mathrm{J} 2000}\right)=\left(100.17^{\circ}, 17.80^{\circ}\right)$ with a TS value of 114. None of these sources affect the spectral parameters fit for the sources under study but they were included for completeness and to make the residual maps flat. To account for correlations between close-by sources, the normalizations of close-by sources (shown as crosses in Figure 11) and of the Galactic and isotropic diffuse emission were left free in the broad-band and in the bin-by-bin fitting procedure. In the case of IC 443, the Crab was modeled with three components for which the normalization was left free following (34): one for the Crab pulsar, and two for the synchrotron and for the inverse Compton part of the Crab Nebula respectively. The maximum likelihood estimation for spatially and spectrally binned data is performed inside a square region of $20^{\circ} \times 20^{\circ}$ centered on each SNR (corresponding to the regions shown in Figure (1). The spatial model for both SNRs was taken as a uniform disk with an angular radius of $21^{\prime}$ (17). The exact choice of the template has only a small impact on the measurement of the spectrum at low energies, due to the large point-spread function of the instrument (the 68\% containment radius is $6^{\circ}$ at $100 \mathrm{MeV}$ ), while it affects the spectrum above $1 \mathrm{GeV}$ at the $<30 \%$ level (11). 


\section{Systematic errors}

For regions near the Galactic equator, the dominant systematic error at energies below $\sim 1 \mathrm{GeV}$ arises from the uncertainty in the model of the Galactic diffuse emission. To test for this effect on the emission assigned to IC 443 and W44, a range of alternative diffuse models calculated by GALPROP (35) and then adjusted for agreement with the observed diffuse gamma-ray emission within the ROI was used. The GALPROP models were chosen to represent a broad range of the parameters scanned in (36). They were adjusted for overall consistency with the all-sky gamma-ray data in a likelihood fit. When fitting the alternative diffuse models in this analysis, the overall normalization was varied. Eight models were tested: the parameters varied among the models are the radial distribution of the cosmic-ray sources (SNR-like or pulsar-like), the size of the cosmic-ray halo (4 kpc or $10 \mathrm{kpc}$ ) and the spin temperature of atomic hydrogen (150 $\mathrm{K}$ or optically thin). It is not a priori obvious which of these (or the standard) diffuse models are the best descriptions of the data for the particular ROIs under study, but the range of diffuse models is intended to test systematics related to uncertainties in the model for the Galactic diffuse emission. The gray bands in Figure 2 show the systematic error due to uncertainties in the Galactic diffuse emission, derived as the envelope of the broad-band fits for the different models.

An additional important source of systematic errors that needs to be considered when analyzing LAT data at low energies is the effect of energy dispersion. In the standard analysis of LAT data, energy dispersion is typically neglected. As long as the LAT effective collection area is relatively independent of energy (above $\sim 200 \mathrm{MeV}$ ) the bias on the flux in an energy bin that arises from misreconstructing the energies of the events is relatively mild (of the order 1-2\%). However, the combination of energy dispersion and the rapidly changing effective area below $100 \mathrm{MeV}$ for the instrument response functions used in this study (P7SOURCE_V6) does result in biased measurements of flux and spectral index of the source under study. In order to quantify the effects of energy dispersion, simulations of the IC 443 region were performed taking into account the observation conditions of these sources in the real dataset and simulating all nearby sources in the region. These simulations include the effect of energy dispersion. The analysis performed on these simulations was exactly the same as performed on the real data. For the study presented here two effects are important: the low-energy index in the broad-band fit $\left(\Gamma_{1}\right)$ will be less steeply falling when going to lower energies and the flux points below $\sim 150$ $\mathrm{MeV}$ will be shifted upwards when ignoring energy dispersion. The simulations can be used to quantify the effect when ignoring energy dispersion. For the spectrum of IC $443, \Gamma_{1}$ is typically less steeply falling by $\sim 1$ (see Figure $\underline{\text { S1) }}$ ) when ignoring the effect. The flux points are higher by the following fractions $(60-81 \mathrm{MeV}: 39 \%, 81-110 \mathrm{MeV}: 23 \%, 110-150 \mathrm{MeV}: 12 \%)$. For W44 we expect corrections of the same magnitude. At higher energies these factors become smaller than 5\%. These studies demonstrate that taking energy dispersion in the analysis into account results in the correct reconstructed values. The magnitude of the bias of the index depends on the spectrum of the Galactic diffuse emission model at energies much lower than the $60 \mathrm{MeV}$ limit for the present analysis. Unlike the simulations just described we do not have 
a perfect model for the diffuse emission, and so even with energy dispersion included in the calculations, $\Gamma_{1}$ is difficult to determine accurately, although for any plausible model the sign of the bias in $\Gamma_{1}$ from neglecting energy dispersion is always the same. In order to not overestimate the magnitude of the spectral break we have decided to neglect energy dispersion in the spectral analysis for this paper. This decision will make the measured low-energy break less pronounced but has little impact on the fitted spectrum for the cosmic-ray protons responsible for the gamma rays, since it just affects the exact shape of the pion bump.

\section{Leptonic models}

In addition to $\pi^{0}$-decay emission, bremsstrahlung and inverse Compton scattering from electrons are possible gamma-ray emission mechanisms for SNRs. Here we consider the cases in which the leptonic processes are dominant in the energy range covered by Fermi-LAT.

As already discussed in the main text, it is difficult to explain the large gamma-ray luminosity of W44 and IC 443 with inverse Compton scattering (see e.g. dashed curve in Figure S2). Considering typical interstellar radiation fields and infrared photons produced locally by the SNR as target photons, the total kinetic energy of electrons is required to be $\sim 10^{51} \mathrm{erg}$, which means almost $100 \%$ of the kinetic energy released by a supernova explosion should be consumed for electron acceleration. In addition, the measurement of a sharply falling spectrum below $\sim 200 \mathrm{MeV}$ is inconsistent with the radio spectrum.

We thus consider models in which electron bremsstrahlung is dominant in the LAT energy band. We assume for the electrons a smoothly broken power law, similar to what was used for the proton spectrum. The radio data of SNRs IC 443 and W44 strongly constrain the electron indices below the break $\left(s_{1}\right)$. On the other hand, the index above the break $\left(s_{2}\right)$ and the location of the break $p_{\text {br }}$ can be determined by fitting the gamma-ray spectral shapes. The total number of electrons, magnetic field strength, and ambient gas density are then chosen to simultaneously fit the synchrotron radio and the gamma-ray bremsstrahlung flux. The magnetic field cannot be too low otherwise a break in the synchrotron spectrum corresponding to $p_{b r}$ appears in the radio band where pure power-law-type spectra are observed. Figures $\mathrm{S2}$ and $\mathrm{S3}$ show the models for IC 443 and W44, respectively (dashed lines). An important discrepancy can be found in the energy range below $<200 \mathrm{MeV}$, where we found low-energy breaks in the Fermi-LAT data.

In order to match the Fermi-LAT data, the electron spectra need to have additional lowenergy breaks. In Figures $\mathbf{S} 2$ and $[\mathbf{S} 3$, we plot bremsstrahlung models with the low-energy breaks added to the electron spectra (dash-dotted lines). To make the bremsstrahlung spectra below $\sim 200 \mathrm{MeV}$ as hard as possible, we have applied abrupt breaks at $300 \mathrm{MeV} c^{-1}$ in the electron spectra for both W44 and IC 443. Even with this extreme assumption, the model curves do not become as hard as the Fermi-LAT spectra below $\sim 200 \mathrm{MeV}$ as seen in Figure 2, although the model and data are marginally consistent considering the systematic errors.

Our results indicate most of the $\mathrm{GeV}$ gamma-ray emission is of hadronic origin, i.e., $\pi^{0}$ decay gamma rays (solid lines). The observed synchrotron radio emission still constrains the accelerated electrons in the SNRs. As shown in Figures $[\mathbf{S} 2$ and $\mathbf{S 3}$, the radio spectra, measured 
at frequencies from several $10 \mathrm{MHz}$ up to $10 \mathrm{GHz}$, have a power-law shape with spectral indices of $\alpha=0.36$ and 0.37 for IC 443 and W44, respectively. This means that the radio-emitting electrons have a power-law index of $s_{1}=1.72$ and 1.74 for IC 443 and W44, respectively. The electron indices are smaller than the proton indices of $s_{1}=2.3-2.4$ determined from the gamma-ray spectra. It should be noted that the radio-emitting electrons have lower momentum compared with the GeV-emitting protons in the crushed cloud scenario of $(20,25)$, and therefore different indices would be explained by introducing another spectral break. Moreover, momentum spectra of electrons and protons do not have the same shape at low energies if re-acceleration of the Galactic cosmic rays is responsible for the observed radio and gamma-ray emission (25). Given that middle-aged, partially radiative SNRs with molecular cloud interactions are complex systems, there is another possibility that the radio-emitting electrons and $\mathrm{GeV}$-emitting protons have different origins, particularly in the case of IC 443. Strong radio emission in IC 443 comes from the northeastern part of the remnant where the shock is propagating in atomic clouds, while the gamma-ray peaks are located near the interacting molecular clouds.

\section{Energetics}

As discussed in the main text, the dominant sites of gamma-ray emission are likely to be the shocked molecular clouds. The mass of the shocked gas is estimated as $M_{\text {shocked }} \sim 1 \times 10^{3} M_{\odot}$ and $\sim 5 \times 10^{3} M_{\odot}$ for IC 443 and W44, respectively. To explain the gamma-ray luminosity, the CR energy density in the clouds should be $\sim 400 \mathrm{eV} \mathrm{cm}^{-3}$, much larger than that of the Galactic CRs. Therefore, adiabatic compression of the Galactic CRs alone cannot explain the required energy density; we need re-acceleration of the pre-existing Galactic CRs (25) and/or freshly accelerated particles that have entered the dense region (20). The total energy content in protons with $p \geq 0.8 \mathrm{GeV}^{-1}$ (above the pion production threshold) amounts to $W_{\mathrm{CR}} \simeq 4 \times$ $10^{49}\left(n / 20 \mathrm{~cm}^{-3}\right)^{-1} \mathrm{erg}$ and $4 \times 10^{49}\left(\mathrm{n} / 100 \mathrm{~cm}^{-3}\right)^{-1} \mathrm{erg}$ for IC 443 and for W44, respectively. Here $n$ denotes the effective gas number density, given by $n=M_{\text {shocked }} / V_{\text {shell }}$ where $V_{\text {shell }}$ is the volume of the SNR shell that contains shock-accelerated CR particles. We assume that the measured gamma-ray emission is produced mainly by CRs with spectra given by Eq. (1) interacting with the shocked clouds inside SNRs. Also, the CR density is assumed to be uniform in the SNR shell, which contains the shocked clouds. Comparing estimates of the explosion energies ( $W_{\mathrm{SN}} \sim 1 \times 10^{51} \mathrm{ergs}$ for IC 443 (37) and $\sim 5 \times 10^{51}$ ergs for W44 (38)) with $W_{\mathrm{CR}}$, we obtain $W_{\mathrm{CR}} / W_{\mathrm{SN}}$ of the order of $1-10 \%$ in these objects at their current ages. The ratio strongly depends on the (rather uncertain) assumed density at the location of the gamma-ray production. 

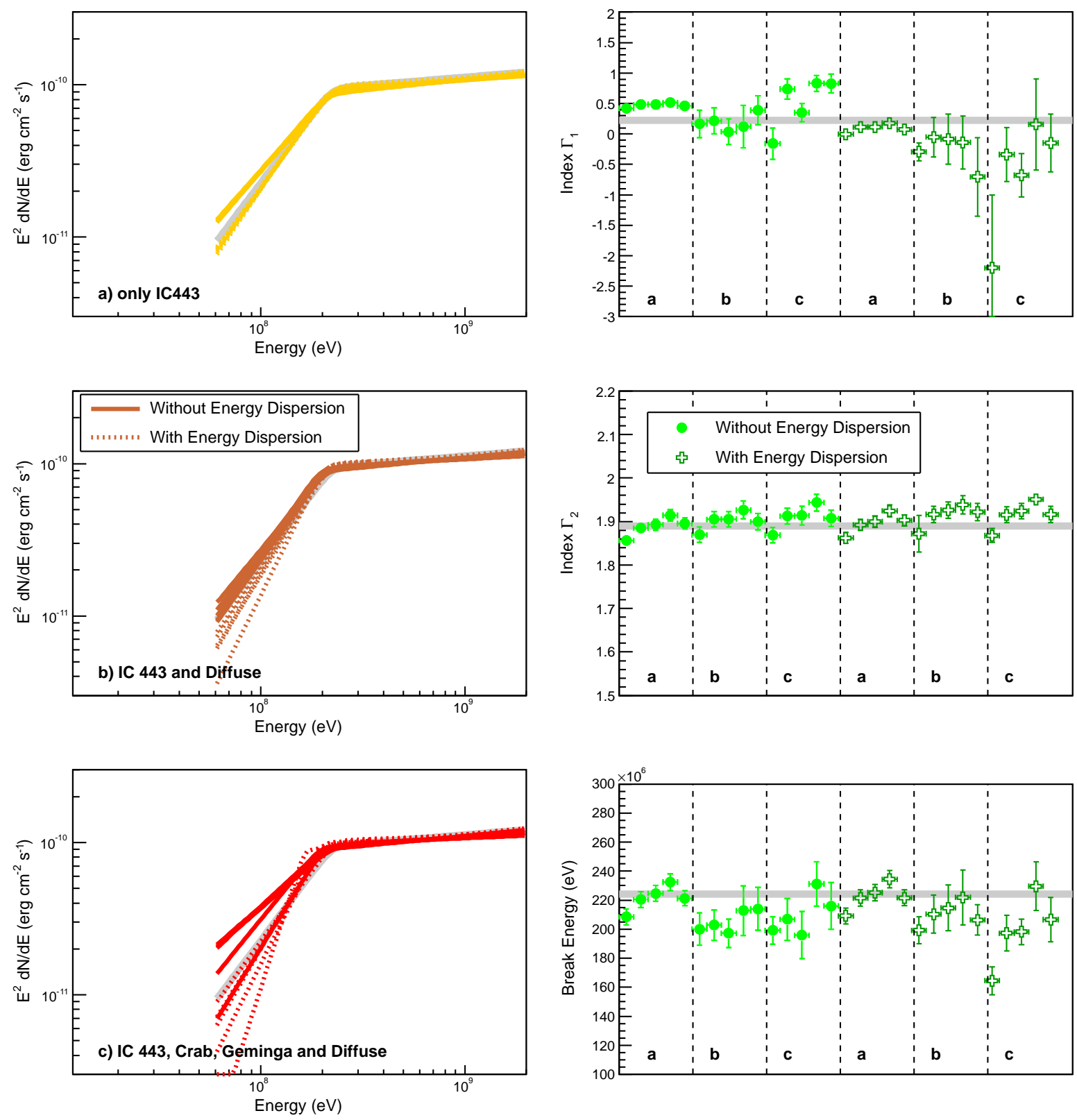

Figure S1: The effect of ignoring or taking the energy dispersion into account in the fitting of simulated data (with energy dispersion) is demonstrated here. For each case 5 realizations of the IC 443 ROI were simulated. The results from fitting with the energy dispersion ignored are shown as solid lines, the results from fitting with the energy dispersion taken into account are shown as dotted lines. The gray lines show the input spectrum. The left panel shows a simulation of IC 443 only, the middle panel a simulation with IC 443 and the Galactic and isotropic diffuse and the right panel a simulation with IC 443, Crab and Geminga and the Galactic and isotropic diffuse emission. The lower row shows the deviations from the input spectrum in the reconstructed values of $\Gamma_{1}, \Gamma_{2}$ and the break energy $E_{\mathrm{br}}$. The solid points show the cases in which the energy dispersion was ignored, the open crosses the cases in which the energy dispersion was switched on. As can be seen, the main effect is on $\Gamma_{1}$ as expected which is $\sim 1$ steeper if energy dispersion is taken into account. 


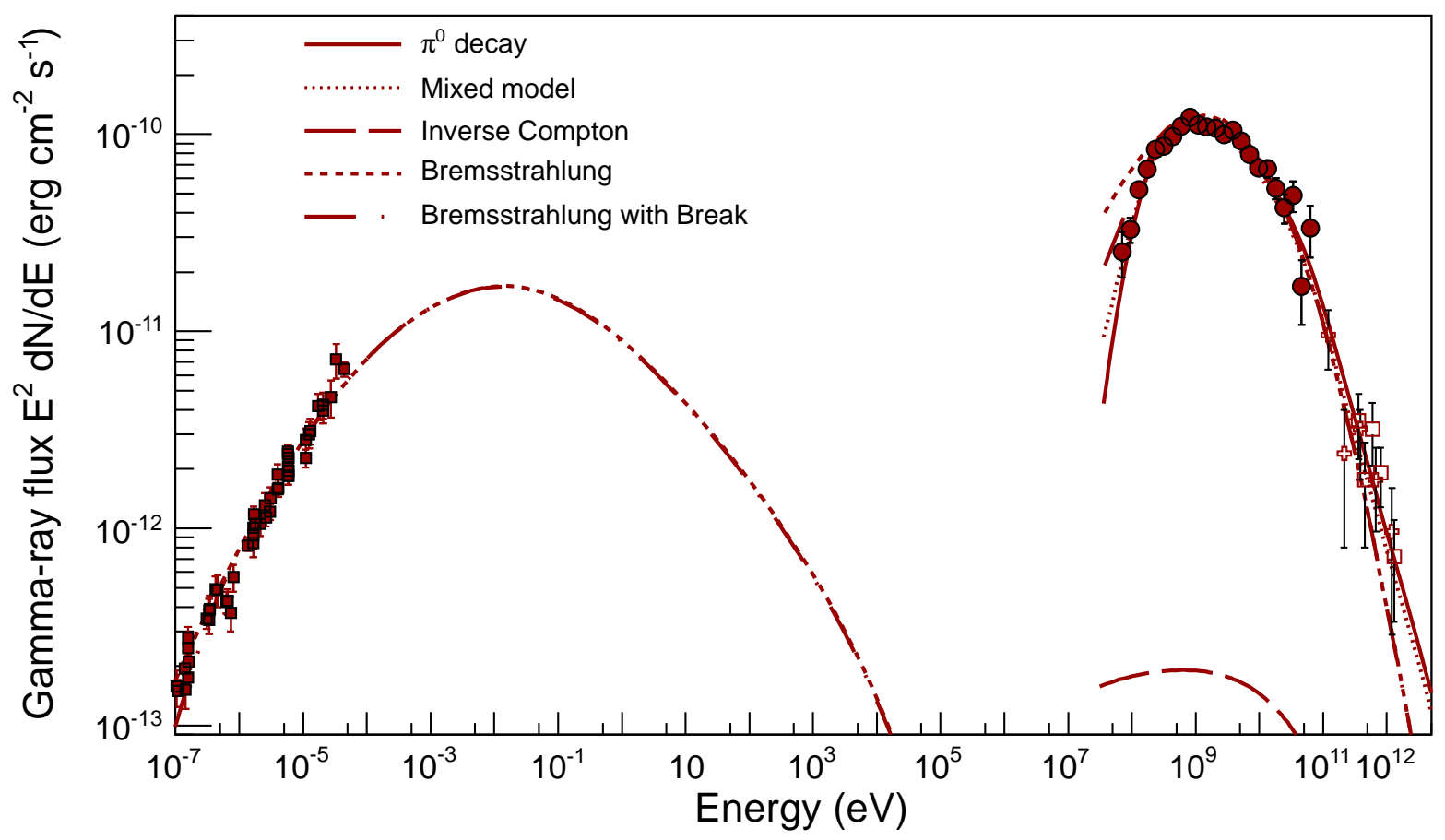

Figure S2: The spectral energy distribution of IC 443 with a model in which electron bremsstrahlung is the dominant radiation process in the gamma-ray band (dashed line and dash-dotted as in Figure 2 ). In the radio band the dominant radiation process is synchrotron emission. The parameters used in the calculation for the dashed curve are $s_{1}=1.72, s_{2}=3.2, p_{\mathrm{br}}=10 \mathrm{GeV} c^{-1}, B=50 \mu \mathrm{G}$, $n=300 \mathrm{~cm}^{-3}$, and $W_{e}=5 \times 10^{47} \mathrm{erg}\left(>1 \mathrm{GeV} \mathrm{c} c^{-1}\right)$. The dash-dotted curve indicates the same model but with an abrupt low-energy break in the electron spectrum at $300 \mathrm{MeV} c^{-1}$. For comparison the best-fit pion-decay model is shown as a solid line. The dotted line shows a combined bremsstrahlung and pion-decay model in which $K_{e p}=0.01$ to demonstrate that such a model is consistent with the data. Radio data are taken from (39) 


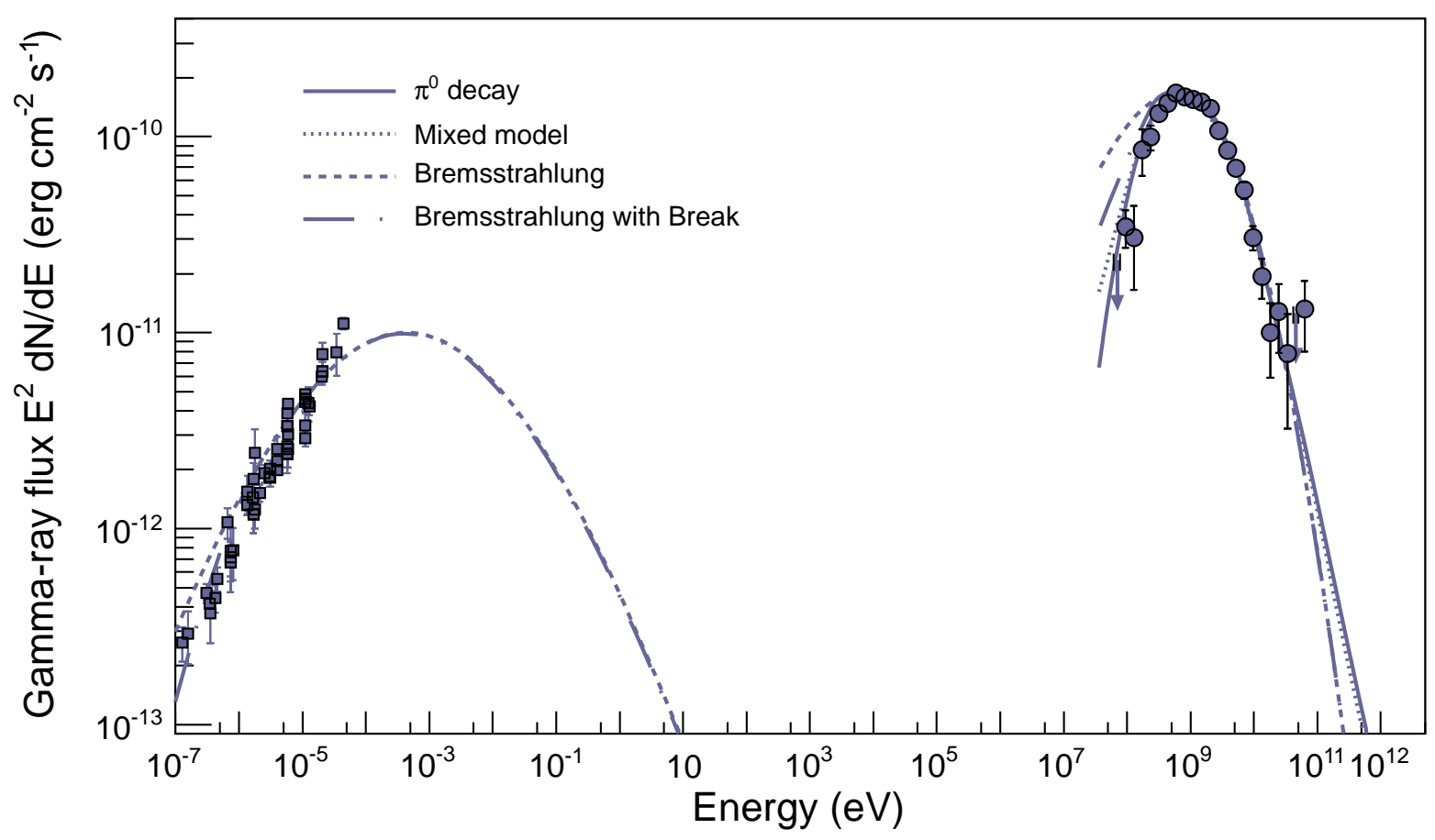

Figure S3: The same as Figure $\underline{\mathrm{S} 2}$ but for W44. The parameters are $s_{1}=1.74, s_{2}=3.7, p_{\mathrm{br}}=$ $10 \mathrm{GeV} c^{-1}, B=90 \mu \mathrm{G}, n=650 \mathrm{~cm}^{-3}$, and $W_{e}=6 \times 10^{47} \mathrm{erg}\left(>1 \mathrm{GeV} \mathrm{c} c^{-1}\right)$. Radio data are taken from (26). 